\title{
Post traumatic anterior angulation of the distal tibia following malunion: A case report of management in a resource constrained setting
}

\author{
E. M. Dim ${ }^{1}$, J.C. Itie' ${ }^{2}$, U.C. Inyang ${ }^{1}$, A. Nwashindi' ${ }^{1}$ M.E. Ugbeye ${ }^{3}$, U.M. Dim ${ }^{4}$ \\ ${ }^{1}$ University of Uyo Teaching Hospital, Uyo, Nigeria, ${ }^{2}$ Department of Surgery, Ladoke Akintola University of Technology Teaching \\ Hospital, Ogbomoso, Nigeria, ${ }^{3}$ Department of Trauma and Orthopaedic Surgery, National Orthopaedic Hospital, Igbobi, Lagos, Nigeria, \\ ${ }^{4}$ Nursing Services Division, National Orthopaedic Hospital, Lagos, Nigeria
}

\section{A B S TR A C T}

Fractures of the shaft of the tibia and fibula are common. Complications are minimal following careful application of principles during treatment by the Orthopaedic Surgeon. However, in our environment, not all patients present to the Orthopaedic Surgeon as they opt for some form of unconventional treatment, usually with the traditional bone setters. Such treatments sometimes lead to clinically unacceptable outcomes. This is a case report of a 42-year old female Nigerian with a 4-year history of post traumatic anterior angulation of the distal right tibia with severely contracted Achilles tendon following traditional bone setter's treatment for open fractures of the distal tibia and fibula. There was $7 \mathrm{~cm}$ of right lower limb shortening and 45 degrees anterior angulation of a tibial malunion. She had a two- stage operation to correct the deformities. Patient achieved a plantigrade foot and fracture union without limb length discrepancy.

Key words: Traditional bone setter, Distal tibial malunion, Anterior angulation, Elongation of Tendo Achilles, Resource constraint
Access this article online

Website:

http://nepjol.info/index.php/AJMS

\section{INTRODUCTION}

The tibia shaft is one of the most commonly fractured long bones in the body. A relatively frequent complication is malunion, which is defined as a deviation from the normal anatomic configuration following healing of a fracture. This implies shortening, angular malalignment or rotational deformities. ${ }^{1,2}$ Treatment goals for tibial fractures, like other long bone fractures, include timely healing, restoration of normal anatomy and functional rehabilitation. Deformity that develops during this healing process results in malunion and may be any combination of translation and rotation in three planes with several factors contributing to this. Malalignment may or may not affect the outcome of a patient who has a tibial shaft fracture, but generally, patients can tolerate angulation of 5 degrees to 8 degrees, malrotation of 15 degrees to 20 degrees and shortening of $2 \mathrm{~cm}^{2}$
Post traumatic angulation following malunion of the distal tibia fractures presents the patient with a twin problem of cosmetic and functional derangements. It is a challenge to the Surgeon, especially in the presence of a precarious soft tissue envelop from poorly managed concomitant open injuries. Deep soft tissue scarring and contracture of the Tendo Achilles, in the case of distal tibia malunion, are other sources of management challenge for the Surgeon. Advances in operative treatment have provided the much needed solution to the bony and soft tissue challenges of post traumatic lower limb deformities. Surgical options include immediate correction by osteotomy and fixation, distraction histiogenesis using external fixation with corticotomy to achieve progressive correction. In rare cases with severe deformity and significant local or systemic co-morbidities, amputation also should be considered as a mode of treatment. ${ }^{2-4}$ 
The major complications of malunion surgery, regardless of technique, include failure to achieve or maintain correction, wound breakdown and infection, neurovascular injury and nonunion.

In resource constrained settings like ours, in spite of advances in operative techniques, the bony and soft tissue challenges of post traumatic lower limb deformities still persist to a large extent, and the consideration of the dangers of soft-tissue compromise limits the options for acute correction and internal fixation.

The aim of this paper is to report the role of Achilles tendon elongation to enhance soft tissue distraction and fracture reduction after osteoclasis of distal tibial malunion with an anterior angulation in a 42 year old female Nigerian.

\section{CASE REPORT}

A 42-year old Nigerian woman presented to us with a four-year history of deformity of her distal right leg and difficulty in walking. She used bilateral axillary crutches. She was involved in a road traffic accident while she was a passenger on a commercial motorcycle that was hit by a car. She sustained an open injury to her right leg and was treated by the traditional bone setters.

On examination, she had a short limb gait with a severe equinus deformity of the right foot. There was an extensive unstable scarring of the skin of the distal right leg covering the antero-medial and antero-lateral surfaces of the leg. There was also a clinically malunited fractures of the distal right tibia and fibula with an anterior angulation of the leg measuring 45 degrees on the goniometer. There was a limb length discrepancy of $7 \mathrm{~cm}$ on the right relative to the left. She had no distal neurovascular deficit (Figure 1).

Antero-posterior and lateral radiographs of the leg showed malunited fractures of the distal tibia and fibula with anterior angulation (Figure 2).

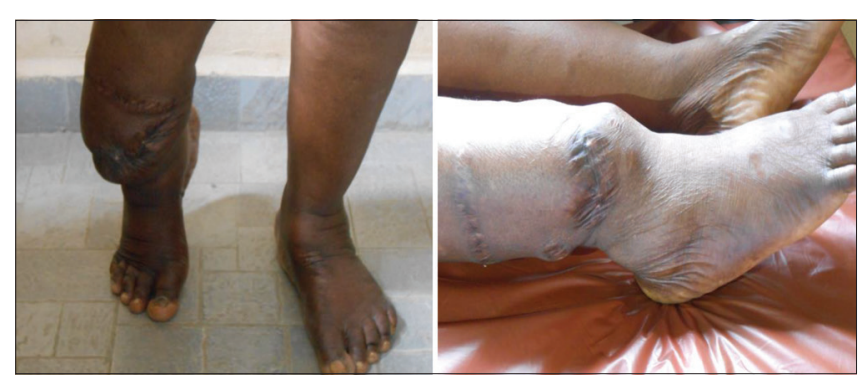

Figure 1: Anterior angulation and scarring of the distal right leg with severe equinus deformity of the foot
The patient had a two-stage operation to correct the deformities. The first stage operation comprised fibula osteotomy through a lateral longitudinal incision over the fibula, osteoclasis of the tibial malunion through an antero-lateral approach and elongation of the Achilles tendon through a posterior incision directly over the tendon (Figure 3a). The intraoperative findings were malunited tibia fracture with $5 \mathrm{~cm}$ overlap of the fragments and anterior angulation, as well as a cross union between the distal fibula fragment and the tibia. The Achilles tendon and the posterior soft tissue of the distal leg were markedly contracted and shortened. It was not possible to reduce the tibial fracture fragments to anatomic position. A distal tibia Steinmann pin was inserted for traction using $3 \mathrm{~kg}$ weight (Figure 3b).

The second stage operation was planned to be an internal fixation with narrow dynamic compression plate, but this

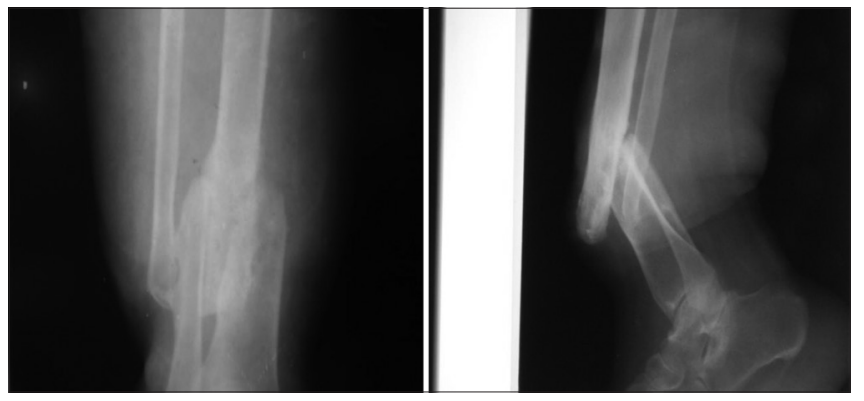

Figure 2: An antero-posterior and lateral radiographs of the leg showing malunited distal tibia and fibula fractures

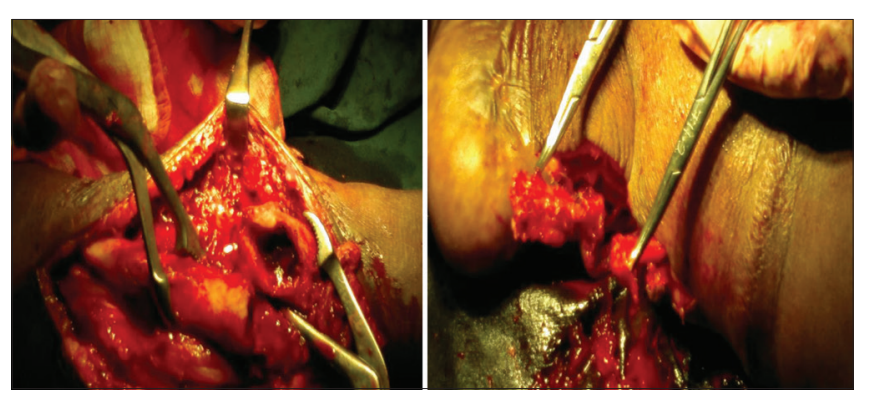

Figure 3a: Osteoclasis of the tibial malunion and elongation of the Achilles Tendon
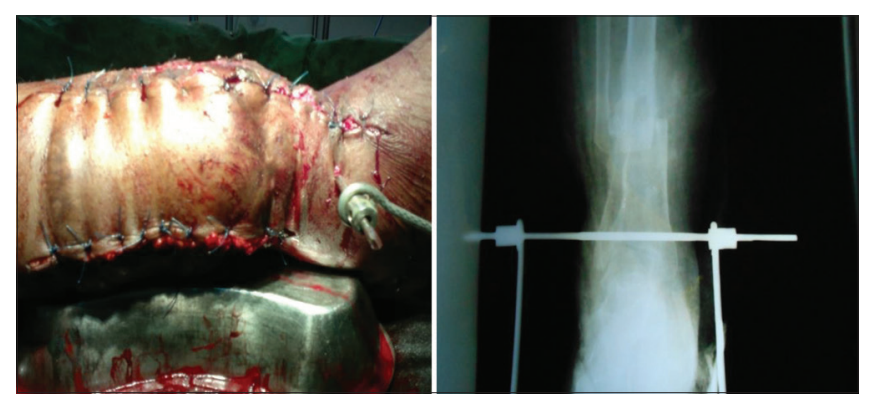

Figure $3 \mathrm{~b}$ : End of first stage operation and an anteroposterior post operative radiograph 
plan was foreclosed by the breakdown of the anterior soft tissue envelope of the leg after the first surgery. The fracture was instead stabilized by means of an external fixator two weeks after the first stage operation and skeletal traction (Figure 4). The patient had wound care post operatively, but declined wound resurfacing by splitthickness skin graft due to financial constraint. The wound was allowed to heal by contraction before the patient was discharged on the $9^{\text {th }}$ week post application of external fixator. The external fixator was removed at this stage and an above the knee scotch cast was applied with the foot achieving plantigrade position (Figure 5). A window on the cast over the residual wound was necessary for alternate day wound care until the wound was completely contracted. There was no residual limb length inequality. The patient was subsequently followed up in the outpatient clinic using radiographs to monitor fracture healing. At 31 weeks post operatively, there was clinico-radiologic evidence of union and the patient was commenced on weight bearing (Figure 6). She was subsequently lost to follow up, and attempts to recall her using her telephone number were not successful.

\section{DISCUSSION}

The index case demonstrates that post traumatic angulation following malunion of the distal tibia fractures can present the patient with a twin problem of cosmetic and functional derangements. The presence of a precarious soft tissue

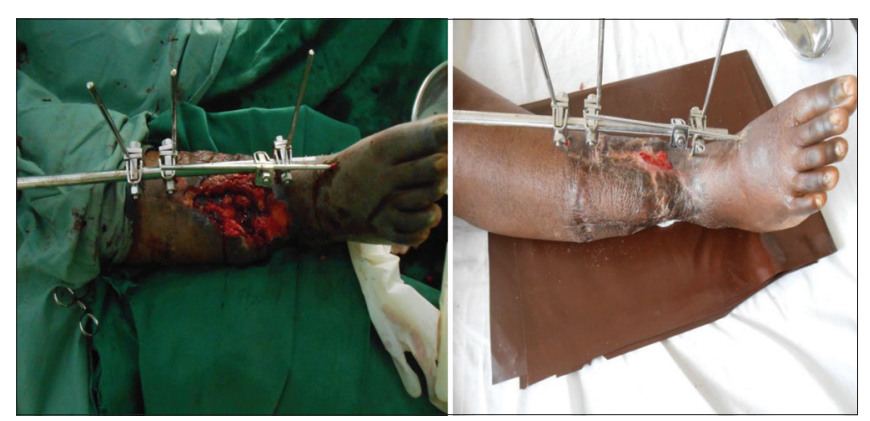

Figure 4: Application of an external fixator at the second surgery (left); the wound after 9 weeks (right)

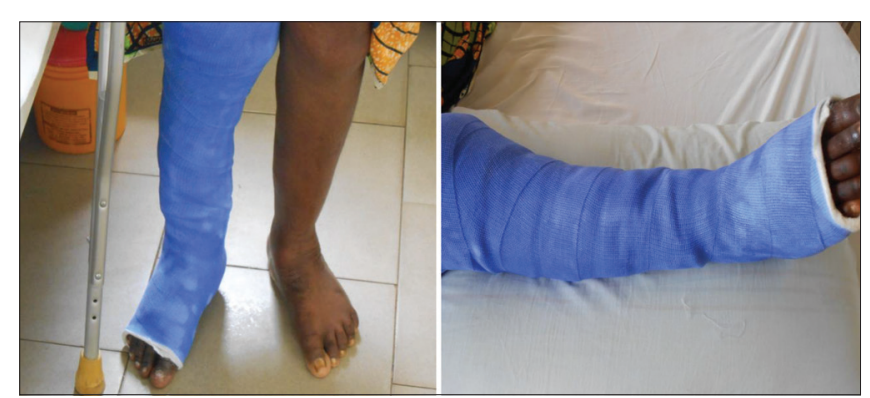

Figure 5: The patient before discharge at 9 weeks post external fixation (Standing and supine) envelop from poorly managed concomitant open injuries, deep soft tissue scarring and contracture of the Tendo Achilles, as we found in this patient, were sources of challenge in the management of this case. These factors were largely responsible for the failure of fracture reduction and primary internal fixation after osteoclasis at the first stage of the operation.

The choice of fixation technique for the correction of a tibial malunion is influenced by the degree of deformity, the presence of a limb-length discrepancy, the condition of the soft tissue envelope, and the experience of the surgeon., ${ }^{2,46}$ Several methods have been described including oblique osteotomy with lag screw and neutralization plate fixation. Opening, closing, neutral wedge or dome osteotomy and fixation with intramedullary nailing have also been used. External fixation devices with distraction osteogenesis have also been used, but deformity correction using external fixation can be difficult. The Ilizarov technique, however, is a satisfactory form of external fixation but many patients refuse this method of treatment because of the discomfort associated with distraction osteogenesis, the duration for which the frame must be applied and frequent postoperative visits. ${ }^{4,5}$ In a study by Ikpeme et $\mathrm{al}^{6}$ on long bone nonunions and malunions in Southern Nigeria, plate and screw osteosynthesis with bone graft augmentation was the commonest treatment modality and the overall union rate was $94 \%$. Primary plate and screw osteosynthesis was not considered a good option in our patient for reasons already mentioned. On the other hand, the Ilizarov technique was an ideal treatment option for her, but this technique is not available in our centre. Our own method of achieving the needed soft tissue distraction in this patient was a combination of elongation of the Achilles tendon at the first stage operation and trans-distal tibia skeletal traction with $3 \mathrm{~kg}$ weight. This technique was to ensure gradual soft tissue stretching and obviate incidental neurovascular injury. Achilles tendon contracture and other soft tissue contractures around the fracture were products of the longstanding nature of the index case. These contractures can significantly affect the outcome of surgery, if not addressed concomitantly with correction of osseous deformity. ${ }^{2}$

We found this technique successful, because the tibia fracture fragments were easily reduced at the second stage operation, after two weeks of skeletal traction. Although the fracture was easily reduced at this time, the intended plate and screw fixation was abandoned for the uni-planer external fixation because of the necrosis and breakdown of the wound and surrounding soft tissue following the first stage operation. Post-operative wound breakdown following corrective surgery for tibial malunion is a noted complication. ${ }^{2}$ The unavailability of the linear rail system or the circular frames for the Ilizarov technique underscores the peculiarity of 
the poor and resource constrained setting of our practice. This picture is further captured by the fact that our patient declined split- thickness skin grafting to resurface her wound because she could not afford to pay for the procedure. We were, therefore constrained to continue wound care with honey dressing at one time and then normal saline at some other point, until healing was achieved by secondary intention. Weight bearing was commenced at 31 weeks when clinico-radiologic evidence of fracture union was established and our patient had no limb length discrepancy.

\section{CONCLUSION}

Achilles Tendon elongation is a useful procedure to aid soft tissue distraction and fracture reduction after osteoclasis of distal tibial malunion with severe anterior angulation.

\section{REFERENCES}

1. Leach RE. Fractures of the tibia and fibula. In: by Rockwood CA $\mathrm{Jr}$, Green DP, eds. Fractures in adults. 2nd ed. Philadelphia: JB Lippincott, 1984, vol2, pp 1593-1663.

2. Mechrefe AP, Koh EY, Trafton PG and DiGiovanni CW. Tibial Malunion. Foot Ankle Clin N Am 2006; 11:19-33.

3. Paley D, Chaudray M, Pirone AM, Lentz $P$ and Kautz D. Treatment of malunions and mal-nonunions of the femur and tibia by detailed preoperative planning and the llizarov techniques. Orthop Clin North Am 1990; 21: 667-691.

4. Sanders R, Anglen JO and Mark JB. Oblique osteotomy for correction of tibial malunion. J Bone Joint Surg Am 1995; 77:240-246.

5. Phieffer LS and Goulet JA. Delayed unions of the tibia. J Bone Joint Surg Am 2006; 88:206-216.

6. Ikpeme IA, Mkpanam NE, Abang IE, Ngim NE and Udosen AM. Long bone non-unions and malunions: Risk factors and treatment outcomes in Calabar, Southern Nigeria. Open Journal of Orthopaedics 2013; 3:253-257.

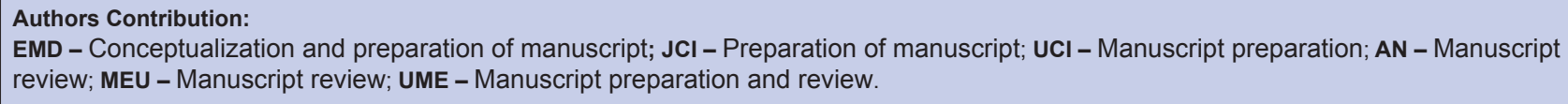

